\title{
Elliptic fibrations on K3 surfaces and Salem numbers of maximal degree
}

\author{
By Xun Yu
}

(Received Aug. 24, 2016)

(Revised Jan. 20, 2017)

\begin{abstract}
We study the maximal Salem degree of automorphisms of K3 surfaces via elliptic fibrations. In particular, we establish a characterization of such maximum in terms of elliptic fibrations with infinite automorphism groups. As an application, we show that any supersingular K3 surface in odd characteristic has an automorphism the entropy of which is the natural logarithm of a Salem number of degree 22.
\end{abstract}

\section{Introduction.}

Let $X$ be a K3 surface defined over an algebraically closed field $k$ of characteristic $p \geq 0$, that is, $X$ is a smooth projective surface defined over $k$ such that $H^{1}\left(X, \mathcal{O}_{X}\right)=0$ and the dualizing sheaf is trivial : $\omega_{X} \simeq \mathcal{O}_{X}$. We denote by $\operatorname{NS}(X)$ the Néron-Severi group of $X$.

The entropy of an automorphism $f: X \longrightarrow X$ is the logarithm of the maximal absolute value of the eigenvalues of the action $f^{*}$ on $\operatorname{NS}(X)$ induced by $f$. This definition is consistent with the topological entropy of automorphisms of smooth complex projective surfaces $([\mathbf{E S 1 3}])$. One knows that the entropy of any automorphism of $X$ is either 0 or the logarithm of a Salem number, and we define Salem degree of $f$ to be 0 or the degree of the Salem number respectively (see Section 3). The maximal Salem degree of automorphisms of $X$ is closely related to both complexity and richness of such automorphisms. Thus, a good understanding of such maximum is of interest. The main results of this note are Theorems 1.1, 1.2, and 1.3.

As in [Ni14], we say an elliptic fibration on $X$ is an elliptic fibration with infinite automorphism group if the set of all automorphisms of $X$ which preserve this fibration is infinite (see Section 2.2). By generalizing [EOY16], we establish a characterization of the maximal Salem degree of automorphisms of K3 surfaces in terms of elliptic fibrations with infinite automorphism groups:

THEOREM 1.1. Let $X$ be a K3 surface defined over an algebraically closed field of characteristic $p \neq 2,3$. Suppose $\operatorname{rk}\left(L_{\infty}(X)\right) \geq 2$, where the sublattice $L_{\infty}(X) \subset \mathrm{NS}(X)$ is defined to be generated by the classes of fibers of all the elliptic fibrations with infinite automorphism groups. Let $d=\operatorname{rk}\left(L_{\infty}(X)\right)$. Then

1) If $d$ is even, $\max \{$ Salem degree of $f \mid f \in \operatorname{Aut}(X)\}=d$;

2010 Mathematics Subject Classification. Primary 14J28, 14J50; Secondary 14D06, 14G99.

Key Words and Phrases. K3 surfaces, automorphisms, elliptic fibrations, Salem numbers. 
2) If $d$ is odd, $\max \{$ Salem degree of $f \mid f \in \operatorname{Aut}(X)\}=d-1$.

Using Theorem 1.1, we show the following comparison type theorem:

TheOREM 1.2. Let $X$ and $Y$ be two $K 3$ surfaces of the same Picard number defined over two algebraically closed fields $k$ and $k^{\prime}\left(\operatorname{char}(k), \operatorname{char}\left(k^{\prime}\right) \neq 2,3\right)$. Suppose $\operatorname{NS}(X)$ is isometric to a sublattice of $\mathrm{NS}(Y)$, and suppose $Y$ has at least one elliptic fibration with infinite automorphism group. Then

$\max \{$ Salem degree of $f \mid f \in \operatorname{Aut}(X)\} \geq \max \{$ Salem degree of $f \mid f \in \operatorname{Aut}(Y)\}$.

Recall that a supersingular K3 surface is a K3 surface with Picard number 22, the maximal possible value. In many senses, supersingular K3 surfaces are the most special K3 surfaces. As an interesting application of Theorem 1.2, we shall also prove the following:

THEOREM 1.3. Let $p$ be an odd prime. Let $X$ be a supersingular K3 surface defined over an algebraically closed field of characteristic $p$. Then there is an automorphism $f \in \operatorname{Aut}(X)$ the entropy of which is the logarithm of a Salem number of degree 22.

In particular, those automorphisms are not geometrically liftable to characteristic 0 (see [EO15], [EOY16]). Many people have studied supersingular K3 surface automorphisms of Salem degree 22 in recent years ([BC16], [EO15], [EOY16], [Sh16], [Sch15], [Br15]). More precisely, we summarize previously known results on such automorphisms as follows:

TheOREM 1.4. Let $p$ be a prime number. Let $X$ be a supersingular K3 surface defined over an algebraically closed field of characteristic p. Let $\sigma(X)$ be the Artin invariant of $X$. Then, in the following cases, $X$ has an automorphism the entropy of which is the logarithm of a Salem number of degree 22 :

(1) $\sigma(X)=1$ and $p=2([\mathbf{B C 1 6}])$;

(2) $\sigma(X)=1$ and $p=3([\mathbf{E O 1 5}])$;

(3) $\sigma(X)=1$ and $p=11$ or $>13([\mathbf{E O Y} 16])$;

(4) $\sigma(X)=1$ and $p \in\{5,7,13\}$, or $2 \leq \sigma(X) \leq 9$ and $3 \leq p \leq 7919$, or $\sigma(X)=10$ and $3 \leq p \leq 17389([\mathbf{S h 1 6}])$.

It is known that for any supersingular K3 surface $X$ in odd characteristic, the NéronSeveri group $\mathrm{NS}(X)$ is isometric to a sublattice of the Néron-Severi group of a supersingular K3 surface of Artin invariant one in the same characteristic ([RS78], cf. [Li15]). Thus, by combining Theorem 1.2 and Theorem 1.4, we can prove Theorem 1.3. The author was recently informed that Simon Brandhorst found an alternative proof of Theorems 1.2 and $1.3([\mathbf{B r 1 6}])$, inspired by the first version of this paper (see also [BG16] for a relevant work).

In Section 6, following [Ni14], we introduce the notion of the exceptional sublattice $E(\mathrm{NS}(X)) \subset \mathrm{NS}(X)$. For an elliptic K3 surface $X$, the exceptional sublattice, the set 
of all the elliptic fibrations with infinitie automorphism groups and the maximal Salem degree of automorphisms of $X$ are closely related to each other (Theorems 1.1, 6.1, 6.2). It is hoped that the exploration of such relationships in this note will find interesting applications in future study of K3 surfaces and other related topics.

Acknowledgements. The author would like to thank Professor Keiji Oguiso for valuable discussions and comments.

\section{K3 surfaces and elliptic fibrations.}

In this paper, for the reader's convenience, we give definitions/explanations of the notations needed. In this section, we fix notations on K3 surfaces and elliptic fibrations.

\subsection{K3 surfaces.}

Let $X$ be a K3 surface defined over an algebraically closed field $k$ of characteristic $p \geq 0$. The Néron-Severi group $\operatorname{NS}(X)$ of $X$ is a free abelian group of finite rank which is denoted by $\rho(X)$ and is called the Picard number of $X$. In characteristic $0, \rho(X)$ is at most 20, but, in positive characteristic, the maximal possible value of $\rho(X)$ is 22 . We denote the intersection form on $\operatorname{NS}(X)$ by $(*, * *)$. Note that $(\operatorname{NS}(X),(*, * *))$ is an even hyperbolic lattice (by definition, a lattice is a free abelian group of finite rank with a $\mathbb{Z}$-valued symmetric bilinear form). Since the intersection form $(*, * *)$ is non-degenerate, the dual $\mathrm{NS}(X)^{*}:=\operatorname{Hom}_{\mathbb{Z}}(\mathrm{NS}(X), \mathbb{Z})$ regarded as a subgroup of $\mathrm{NS}(X) \otimes \mathbb{Q}$ contains $\mathrm{NS}(X)$ through this intersection form. The discriminant group of $X$ is defined to be the quotient $\mathrm{NS}(X)^{*} / \mathrm{NS}(X)$.

According to [Ar74], the discriminant group $\mathrm{NS}(X)^{*} / \mathrm{NS}(X)$ of a supersingular K3 surface $X$ is, as an abelian group, isomorphic to $(\mathbb{Z} / p)^{2 \sigma(X)}$, where $\sigma(X)$ is an integer such that $1 \leq \sigma(X) \leq 10$. The integer $\sigma(X)$ is called the Artin invariant of $X$. In each positive characteristic $p>0$, there is, up to isomorphism, a unique Artin invariant one supersingular K3 surface $X(p)([\mathbf{O g u 7 9}]$, [Shi75]).

\subsection{Elliptic fibrations on K3 surfaces.}

Let $X$ be a K3 surface defined over an algebraically closed field $k$ of characteristic $p \neq 2,3$. According to Piatetsky-Shapiro and Shafarevich [PS71], elliptic fibrations on $X$ are in one-to-one correspondence with primitive isotropic nef elements $e \in \mathrm{NS}(X)$. That is, $e \neq 0, e^{2}=0, e / n \in \mathrm{NS}(X)$ only for integers $n= \pm 1, e \cdot D \geq 0$ for any effective divisor $D$ on $X$. For such $e \in \operatorname{NS}(X)$, the complete linear system $|e|$ is one dimensional without base points, and it gives an elliptic fibration $|e|: X \longrightarrow \mathbb{P}^{1}$, that is, the general fiber is a curve of genus 1 .

For any $c \in \mathrm{NS}(X)$, we set

$$
\operatorname{Aut}(X)_{c}:=\left\{f \in \operatorname{Aut}(X) \mid f^{*}(c)=c\right\} .
$$

We introduce some notations related to elliptic fibrations on $X$ :

$\mathcal{E}(X):=\{e \in \mathrm{NS}(X) \mid e$ is primitive, isotropic, and nef $\} ;$

$\mathcal{E}_{\infty}(X):=\left\{e \in \mathcal{E}(X) \mid \operatorname{Aut}(X)_{e}\right.$ is an infinite group $\}$, and we say an elliptic fibration on $X$ is an elliptic fibration with infinite automorphism group if the corresponding class of this fibration is in $\mathcal{E}_{\infty}(X)$; 
The sublattice $L_{\infty}(X) \subset \mathrm{NS}(X)$ is generated by all the elements in $\mathcal{E}_{\infty}(X)$.

For any nonzero isotropic element $c \in \mathrm{NS}(X)$, the sublattice $\left(c^{\perp}\right)^{(2)} \subset c^{\perp}$ is defined to be generated by $c$ and by all elements with square $(-2)$ in $c^{\perp}$, where $c^{\perp}$ is the orthogonal complement to $c$ in $\operatorname{NS}(X)$. The following lemma gives a test of an elliptic fibration with infinite automorphism group:

Lemma 2.1. Let $e \in \mathcal{E}(X)$. Then $e \in \mathcal{E}_{\infty}(X)$ if and only if $\operatorname{rk}\left(e^{\perp}\right)-\operatorname{rk}\left(\left(e^{\perp}\right)^{(2)}\right)>0$.

Proof. We use $\operatorname{Amp}(X) \subset \mathrm{NS}(X) \otimes \mathbb{R}$ to denote the ample cone of $X$. Let

$$
A(\mathrm{NS}(X)):=\{f \in \mathrm{O}(\mathrm{NS}(X)) \mid f(\operatorname{Amp}(X))=\operatorname{Amp}(X)\} .
$$

Let $A(\mathrm{NS}(X))_{e} \subset A(\mathrm{NS}(X))$ be the stabilizer subgroup of $e$. By [LM11, Theorem 6.1], the natural map Aut $(X) \longrightarrow A(\mathrm{NS}(X))$ has finite kernel and cokerel. Then $\operatorname{Aut}(X)_{e}$ and $A(\mathrm{NS}(X))_{e}$ are isomorphic up to finite groups. By [Ni83, Corollary 1.5.4] (notice that the proof of [Ni83, Corollary 1.5.4] works for even hyperbolic lattices, in particular, $\mathrm{NS}(X)$, and is valid in any characteristic), $A(\mathrm{NS}(X))_{e}$ is infinite if and only if $\operatorname{rk}\left(e^{\perp}\right)-$ $\operatorname{rk}\left(\left(e^{\perp}\right)^{(2)}\right)>0$. Thus, $\operatorname{Aut}(X)_{e}$ is infinite if and only if $\operatorname{rk}\left(e^{\perp}\right)-\operatorname{rk}\left(\left(e^{\perp}\right)^{(2)}\right)>0$.

Let $\phi: \operatorname{Aut}(X) \longrightarrow \mathrm{O}(\mathrm{NS}(X))$ be the natural map which sends any $f \in \operatorname{Aut}(X)$ to the induced isometry $f^{*}$ of $\operatorname{NS}(X)$. By definition of $L_{\infty}(X)$, it is clear that $L_{\infty}(X)$ is an $\operatorname{Aut}(X)$-stable sublattice of $\operatorname{NS}(X)$ (i.e., for all $\left.f \in \operatorname{Aut}(X), f^{*}\left(L_{\infty}(X)\right)=L_{\infty}(X)\right)$. Thus $\phi$ naturally induces another map

$$
\psi: \operatorname{Aut}(X) \longrightarrow \mathrm{O}\left(L_{\infty}(X)\right) \times \mathrm{O}\left(L_{\infty}(X)^{\perp}\right)
$$

such that, for any $f \in \operatorname{Aut}(X), \psi(f)=\left(\left.f^{*}\right|_{L_{\infty}(X)},\left.f^{*}\right|_{\left.L_{\infty}(X)^{\perp}\right) \text {. Let }}\right.$

$$
\pi: \mathrm{O}\left(L_{\infty}(X)\right) \times \mathrm{O}\left(L_{\infty}(X)^{\perp}\right) \longrightarrow \mathrm{O}\left(L_{\infty}(X)\right)
$$

be the natural projection map.

Lemma 2.2. Suppose $X$ has at least two different elliptic fibrations with infinite automorphism groups, i.e., $\operatorname{rk}\left(L_{\infty}(X)\right) \geq 2$. Then both $\operatorname{Ker}(\psi)$ and $\operatorname{Ker}(\pi \circ \psi)$ are finite groups.

Proof. Let $d=\operatorname{rk}\left(L_{\infty}(X)\right)$. Since $d \geq 2$, it follows that $L_{\infty}(X)$ is a hyperbolic lattice of signature $(1, d-1)$. Then the orthogonal complement $L_{\infty}(X)^{\perp} \subset \mathrm{NS}(X)$ of $L_{\infty}(X)$ is a negative definite lattice of $\operatorname{rank} \rho(X)-d$. By [LM11, Theorem 6.1], $\operatorname{Ker}(\phi)$ is a finite group. Note that $L_{\infty}(X) \oplus L_{\infty}(X)^{\perp}$ is a sublattice of $\mathrm{NS}(X)$ of finite index. Thus, $\operatorname{Ker}(\psi)$ is equal to $\operatorname{Ker}(\phi)$ and hence is also a finite group. The group $\mathrm{O}\left(L_{\infty}(X)^{\perp}\right)$ is finite because $L_{\infty}(X)^{\perp}$ is negative definite. Since the quotient $\operatorname{Ker}(\pi \circ \psi) / \operatorname{Ker}(\psi)$ is isomorphic to a subgroup of $\mathrm{O}\left(L_{\infty}(X)^{\perp}\right)$, it follows that $\operatorname{Ker}(\pi \circ \psi) / \operatorname{Ker}(\psi)$ is finite. Then $\operatorname{Ker}(\pi \circ \psi)$ is also finite. 


\section{Salem numbers and entropy.}

Let $(L,(*, * *))$ be a hyperbolic lattice, i.e., a pair consisting of a free abelian group of rank $1+t$ and a $\mathbb{Z}$-valued symmetric bilinear form $($,$) on L$ of signature $(1, t)$ with $t>0$. For any ring $K$, we use $L_{K}$ to denote the scalar extension of $L$ to $K$. We fix some notations (they are the same as in [EOY16], but, for the reader's convenience, we repeat here):

$\mathrm{O}(L):=$ the orthogonal group of the lattice $L$

$\mathrm{SO}(L):=\{g \in \mathrm{O}(L) \mid \operatorname{det}(g)=1\}$

$P:=\left\{x \in L_{\mathbb{R}} \mid\left(x^{2}\right)>0\right\}$, notice that $P$ consists of two connected components, say $\pm C$;

$\mathrm{O}^{+}\left(L_{\mathbb{R}}\right):=\{g \in \mathrm{O}(L)(\mathbb{R}) \mid g(C)=C\} ;$

$\mathrm{O}^{+}(L):=\mathrm{O}(L)(\mathbb{Z}) \cap \mathrm{O}^{+}\left(L_{\mathbb{R}}\right)$

$\mathrm{SO}^{+}(L):=\mathrm{O}(L)(\mathbb{Z}) \cap \mathrm{O}^{+}\left(L_{\mathbb{R}}\right) \cap \mathrm{SO}(L)(\mathbb{R})$.

Definition 3.1. A Salem number of degree $2 d$ is a real algebraic integer $a>1$ whose Galois conjugates consist of $1 / a$ and $2 d-2$ complex numbers of modulus 1 . A Salem polynomial is the minimal polynomial of a Salem number.

Salem numbers and isometries of hyperbolic lattices are closely related:

Proposition 3.2 (See $[\mathbf{M c 0 2}],[\mathbf{O g} \mathbf{1 0}])$. Let $f \in \mathrm{O}^{+}(L)$. We denote the characteristic polynomial of $f$ by $p(x)$. Then one of the following two statements is true:

(1) $p(x)$ is the product of cyclotomic polynomials;

(2) $p(x)=c(x) s(x)$, where $c(x)$ is the product of cyclotomic polynomials and $s(x)$ is a Salem polynomial.

Definition 3.3. Let $f \in \mathrm{O}^{+}(L)$. The entropy $h(f)$ of $f$ is defined by

$$
h(f)=\log (r(f)) \geq 0,
$$

where $r(f)$ is the spectral radius of $f$ (i.e., the maximum of the absolute values of the complex eigenvalues of $f$ acting on $L)$.

The next definition can be viewed as a generalization of the topological entropy of automorphisms of smooth complex projective surfaces ([ES13]):

Definition 3.4. Let $S$ be a smooth projective surface, and let $f \in \operatorname{Aut}(S)$. The entropy $h(f)$ of $f$ is defined by

$$
h(f)=\log r\left(f^{*} \mid \mathrm{NS}(X)\right),
$$

where $f^{*}$ is the action on $\operatorname{NS}(X)$ induced by $f$.

Definition 3.5. Let $X$ be a K3 surface, and let $f \in \operatorname{Aut}(X)$. The Salem degree of $f$ is defined by 
Salem degree of $f= \begin{cases}0 & \text { if the entropy } h(f) \text { is } 0, \\ d & \text { if the entropy } h(f) \text { is log of a Salem number of degree } d .\end{cases}$

REMARK 3.6. In order to study the maximal Salem degree of automorphisms of any K3 surface $X$, we will take $L$ to be the sublattice $L_{\infty}(X)$ of the Néron-Severi group $\mathrm{NS}(X)$ and $C$ to be the connected component of $P$ the closure of which contains all elements in $\mathcal{E}_{\infty}(X)$.

\section{A few observations from group theory.}

Theorem 4.1 and Theorem 4.4 are generalizations of [EOY16, Theorem 4.1] and [EOY16, Theorem 4.6]. Proofs are similar. Here, for the reader's convenience, we give the proof of Theorem 4.1 ii) and Theorem 4.4 below.

THEOREM 4.1. Let $L$ be a hyperbolic lattice of rank $d \geq 2$ and $G \subset \mathrm{SO}^{+}(L)$ be a subgroup. Assume that $G$ has no non-trivial $G$-stable $\mathbb{R}$-linear subspace of $L_{\mathbb{R}}$. Then

i) If $d$ is even, then there is an element $g \in G$ such that the characteristic polynomial of $g$ is a Salem polynomial of degree $d$;

ii) If $d$ is odd, then there is an element $g \in G$ such that the characteristic polynomial of $g$ is $(t-1) s(t)$, where $s(t)$ is a Salem polynomial of degree $d-1$.

The case i) is just [EOY16, Theorem 4.1]. The proof of the case ii) is completely similar to that of the case i), and we will sketch it below:

Proof. We assume $d$ is odd. Let $P_{d} \subset \mathbb{Z}[t]$ be the set of monic polynomials of degree $d$. Then $P_{d}$ is identified with the affine variety $\mathbb{A}^{d}$ defined over $\mathbb{Z}$. The map

$$
\text { char : } \mathrm{SO}(L) \rightarrow P_{d}, \quad h \mapsto \Phi_{h}(t):=\operatorname{det}\left(t I_{d}-h\right)
$$

is a morphism of affine varieties. Let $u_{1}(t):=t-1, u_{2}(t):=t+1, \ldots, u_{N}(t)$ be the cyclotomic polynomials in $\mathbb{Z}[t]$ of degree $\leq d$, where $N$ is the cardinarity of the cyclotomic polynomials of degree $\leq d$. The subsets $P_{1}:=\left\{p(t) \in P_{d}(\mathbb{C})\left|u_{1}^{2}(t)\right| p(t)\right\}$ and $P_{i}:=$ $\left\{p(t) \in P_{d}(\mathbb{C})\left|u_{i}(t)\right| p(t)\right\}$ for $i \geq 2$ define proper closed algebraic subvarieties of $P_{d} \otimes_{\mathbb{Z}} \mathbb{Q}$, thus so is their finite union $Q_{d}:=\cup_{i=1}^{N} P_{i} \subset P_{d} \otimes_{\mathbb{Z}} \mathbb{Q}$. (Notice that the sets $P_{i}, i \geq 2$, defined here is the same as those defined in [EOY16]. However, the set $P_{1}$ here is slightly different from that in [EOY16]. This is because, when $d$ is odd, for any $g \in \operatorname{SO}(L)(\mathbb{Z})$, 1 is an eigenvalue of $g$, and hence $t-1$ divides the characteristic polynomial of $g$.)

Let $g \in G$. Its characteristic polynomial $\Phi_{g}(t) \in \mathbb{Z}[t]$ is monic and of degree $d$. By Proposition 3.2, $\Phi_{g}(t)$ is the product of cyclotomic polynomials and of at most one Salem polynomial counted with multiplicities. Thus, $\Phi_{g}(t)$ is divided by a Salem polynomial of degree $d-1$ if and only if $\Phi_{g}(t) \in P_{d}(\mathbb{C}) \backslash Q_{d}$. The following lemma completes the proof:

Lemma 4.2. There is an element $g \in G$ such that $\Phi_{g}(t) \in P_{d}(\mathbb{C}) \backslash Q_{d}$.

Proof. See the proof of [EOY16, Lemma 4.5]. 
This completes the proof of Theorem 4.1.

REMARK 4.3. In order to prove Theorem 1.3, we essentially only need the case i) of Theorem 4.1. However, we need both two cases of Theorem 4.1 to prove Theorem 1.1 which can apply to other K3 surfaces besides supersingular K3 surfaces.

THEOREM 4.4. Let $L$ be a hyperbolic lattice of signature $(1, r+1)$ with $r \geq 0$ and let $e \in L$ be a primitive element such that $(e, e)=0$. Let $g \in \operatorname{SO}(L)(\mathbb{Z})$ be such that $\operatorname{ord}(g)=\infty$ and $g(e)=e$. Suppose $V$ is a g-stable $\mathbb{R}$-linear subspace of $L_{\mathbb{R}}$. Then either $V \subset e^{\perp}$, or $e \in V($ or both $)$.

Proof. $\quad$ Choose a $\mathbb{Q}$-bases of $L_{\mathbb{Q}}$ :

$$
\left\langle e, w_{1}, \ldots, w_{d-2}, u\right\rangle
$$

where $w_{i} \in e^{\perp}$, and $(u, e)=1$.

By [Og09, proof of Lemma 3.6] (see also [EOY16, Lemma 4.7]), replacing $g$ by a suitable power $g^{N}(N>0)$ if necessary, we may assume

$$
g=\left(\begin{array}{ccc}
1 & \mathbf{a}^{t} & c \\
\mathbf{0} & I_{r} & \mathbf{b} \\
0 & \mathbf{0}^{t} & 1
\end{array}\right)
$$

with respect to the $\mathbb{Q}$-bases chosen above. Here $1,0 \in \mathbb{Q}$ are the unit and the zero, $c$ is in $\mathbb{Q}, I_{r}$ is the $r \times r$ identity matrix, $\mathbf{0} \in \mathbb{Q}^{r}$ is the zero vector, $\mathbf{b} \in \mathbb{Q}^{r}$ is a column vector, $\mathbf{a}^{t}$ is the transpose of a column vector $\mathbf{a} \in \mathbb{Q}^{r}$, and simiarly for $\mathbf{0}^{t}$.

We claim that $\mathbf{b} \neq \mathbf{0}$. Suppose otherwise. Then $g(u)=u+c e$, it follows that

$$
(u, u)=(g(u), g(u))=(u+c e, u+c e)=(u, u)+2 c(u, e)=(u, u)+2 c,
$$

which implies $c=0$. Then for all $1 \leq i \leq r$

$$
\left(u, w_{i}\right)=\left(g(u), g\left(w_{i}\right)\right)=\left(u, w_{i}+a_{i} e\right)=\left(u, w_{i}\right)+a_{i}, \text { where } a_{i}=\mathbf{a}^{t} \cdot \mathbf{e}_{i} .
$$

Therefore, $a_{i}=0$ for all $i$. So $g=I d$, a contradiction to $\operatorname{ord}(g)=\infty$. Therefore, $\mathbf{b} \neq \mathbf{0}$.

Without loss of generality, from now on, we may assume $\mathbf{b}=\mathbf{e}_{r}$.

We claim that $a_{r} \neq 0$. Suppose otherwise. Then for all $k \geq 1$,

$$
g^{k}(u)=u+k w_{r}+k c e .
$$

Since $g^{k}$ preserves intersection form, it follows that

$$
(u, u)=\left(g^{k}(u), g^{k}(u)\right)=(u, u)+k^{2}\left(w_{r}, w_{r}\right)+2 k\left(u, w_{r}\right)+2 k c
$$

whence

$$
\left(w_{r}, w_{r}\right) k^{2}+\left(2\left(u, w_{r}\right)+2 c\right) k=0
$$

for all positive integers $k$, a contradiction to $\left(w_{r}, w_{r}\right)<0$. Therefore, $a_{r} \neq 0$. 
If $V \subset e^{\perp}$, then we are done.

From now on, we assume $V$ is not contained in $e^{\perp}$. Then there exists $0 \neq v \in V$ of the following form

$$
v=u+\alpha e+\sum_{i=1}^{r} \beta_{i} w_{i},
$$

where $\alpha, \beta_{i} \in \mathbb{R}$. Then $g(v)=u+w_{r}+(c+\alpha) e+\sum_{i=1}^{r} \beta_{i}\left(w_{i}+a_{i} e\right)$ whence $g(v)-v=$ $w_{r}+c e+\sum_{i=1}^{r} \beta_{i} a_{i} e$. Then $g(g(v)-v)-(g(v)-v)=a_{r} e \in V$ since $V$ is $g$-stable. It follows that $e \in V$ by $a_{r} \neq 0$.

\section{Proofs of Theorems $1.1,1.2$ and 1.3.}

We need the following result to prove Theorem 1.1:

THEOREM 5.1. Let $X$ be a K3 surface defined over an algebraically closed field $k$ of characteristic $p \neq 2,3$. Suppose $\operatorname{rk}\left(L_{\infty}(X)\right) \geq 2$, where the sublattice $L_{\infty}(X) \subset \operatorname{NS}(X)$ is generated by all the elliptic fibrations with infinite automorphism groups. To simplify the notation, we set $L:=L_{\infty}(X)$. Then there exists a subgroup $G \subset \operatorname{Aut}(X)$ such that

1) $G^{\prime} \subset \mathrm{SO}^{+}(L)$, where $G^{\prime}:=(\pi \circ \psi)(G)$ (see Section 2.2 for definition of $\pi$ and $\left.\psi\right)$, and

2) Any $G^{\prime}$-stable $\mathbb{R}$-linear subspace of $L_{\mathbb{R}}$ is either $\{0\}$ or $L_{\mathbb{R}}$.

Proof. In order to construct $G$, we need the following:

Lemma 5.2. For any $e \in \mathcal{E}_{\infty}(X)$, there exists $g_{e} \in \operatorname{Aut}(X)_{e}$ such that $(\pi \circ \psi)\left(g_{e}\right) \in$ $\mathrm{SO}(L)$ and $\operatorname{ord}\left((\pi \circ \psi)\left(g_{e}\right)\right)=\infty$.

Proof. By definition of $\mathcal{E}_{\infty}(X), \operatorname{Aut}(X)_{e}$ is an infinite subgroup of $\operatorname{Aut}(X)$. Since, by Lemma 2.2, $\operatorname{Ker}(\pi \circ \psi) \cap \operatorname{Aut}(X)_{e}$ is finite, the image $(\pi \circ \psi)\left(\operatorname{Aut}(X)_{e}\right)$ is an infinite subgroup of $\mathrm{O}(L)$. Since $e \in L$, by [Og07, Proposition 2.9], every element of $(\pi \circ \psi)\left(\operatorname{Aut}(X)_{e}\right) \cap \mathrm{SO}(L)$ is of null-entropy. Thus, there exists $h \in$ $(\pi \circ \psi)\left(\operatorname{Aut}(X)_{e}\right) \cap \mathrm{SO}(L)$ such that $\operatorname{ord}(h)=\infty$ by [Og07, Proposition $\left.2.2(3)\right]$ (notice that the proof of [Og07, Proposition $2.2(3)]$ is based on even hyperbolic lattices, and is valid in any characteristic). Choose any $g_{e} \in(\pi \circ \psi)^{-1}(h)$. Then order ord $\left(g_{e}\right)=\infty$ and $(\pi \circ \psi)\left(g_{e}\right) \in \mathrm{SO}(L)$.

Now, for any $e \in \mathcal{E}_{\infty}(X)$, by Lemma 5.2, we can choose some $g_{e} \in \operatorname{Aut}(X)_{e}$ such that $(\pi \circ \psi)\left(g_{e}\right) \in \mathrm{SO}(L)$ and $\operatorname{ord}\left((\pi \circ \psi)\left(g_{e}\right)\right)=\infty$. We set $G:=$ the subgroup of $\operatorname{Aut}(X)$ generated by $\left\{g_{e} \mid e \in \mathcal{E}_{\infty}(X)\right\}$. Let $G^{\prime}:=(\pi \circ \psi)(G)$. Then $G^{\prime} \subset \mathrm{SO}(L)$. Since $G^{\prime}\left(\mathcal{E}_{\infty}(X)\right)=\mathcal{E}_{\infty}(X)$, it follows that $G^{\prime} \subset \mathrm{SO}^{+}(L)$, which is the statement 1$)$.

Next we prove the statement 2$)$. Let $V$ be a non-zero $G^{\prime}$-stable $\mathbb{R}$-linear subspace of $L_{\mathbb{R}}$. We may assume $V \neq L_{\mathbb{R}}$ (otherwise, we are done). Then there exists $e_{0} \in \mathcal{E}_{\infty}(X)$ such that $e_{0} \notin V$. Since $V$ is $G^{\prime}$-stable, it follows that $V$ is also $(\pi \circ \psi)\left(g_{e_{0}}\right)$-stable. Then by Theorem 4.4, we have that $V \subset e_{0}^{\perp}$. For any $e^{\prime} \in \mathcal{E}_{\infty}(X)$ such that $e^{\prime} \neq e_{0}$, by Hodge-index Theorem, we have that $\left(e_{0}, e^{\prime}\right)>0$, which implies $e^{\prime} \notin V$. Then by 
Theorem 4.4 again, $V \subset e^{\perp \perp}$. Therefore, $V \subset \cap_{e \in \mathcal{E}_{\infty}(X)} e^{\perp}$. But $\mathbb{R}\left\langle\mathcal{E}_{\infty}(X)\right\rangle=L_{\mathbb{R}}$ and $L$ is a non-degenerate lattice. Thus $V=0$. This completes the proof of Theorem 5.1.

Proof of Theorem 1.1. We prove the case 1) and the proof for the case 2) is similar.

Suppose $d$ is even. Let $L=L_{\infty}(X)$. Since $d \geq 2$, it follows that the orthogonal complement $L^{\perp}$ of $L$ in $\operatorname{NS}(X)$ is negative definite. Note that, for any $f \in \operatorname{Aut}(X)$, we have $f^{*}(L)=L$ and $f^{*}\left(L^{\perp}\right)=L^{\perp}$. So the Salem degree of any automorphism of $X$ is an even integer $\leq d$. Thus, $\max \{$ Salem degree of $f \mid f \in \operatorname{Aut}(X)\} \leq d$. On the other hand, by Theorem 4.1 and Theorem 5.1, $X$ has an automorphism the entropy of which is a Salem number of degree $d$, which implies $\max \{$ Salem degree of $f \mid f \in \operatorname{Aut}(X)\} \geq d$. Therefore, $\max \{$ Salem degree of $f \mid f \in \operatorname{Aut}(X)\}=d$.

Proof of Theorem 1.2. If $Y$ has exactly one elliptic fibration with infinite automorphism group (i.e., $\operatorname{rk}\left(L_{\infty}(Y)\right)=1$ ), then every automorphism of $Y$ must preserve this elliptic fibration. Then, by [Og07, Proposition 2.9] (again the proof of [Og07, Proposition 2.9] is valid in any characteristic), the entropy of any automorphism of $Y$ must be zero, which implies $\max \{$ Salem degree of $f \mid f \in \operatorname{Aut}(Y)\}=0$ (thus the conclusion of Theorem 1.2 is true).

Therefore, we may assume $Y$ has at least two elliptic fibrations with infinite automorphism groups. Then by Theorem 1.1, it suffices to prove $\operatorname{rk}\left(L_{\infty}(X)\right) \geq \operatorname{rk}\left(L_{\infty}(Y)\right)$.

Fix an isometric embedding $\iota: \operatorname{NS}(X) \hookrightarrow \operatorname{NS}(Y)$. Let $d=\operatorname{rk}\left(L_{\infty}(Y)\right)$. Then we can choose $e_{1}, \ldots, e_{d} \in \mathcal{E}_{\infty}(Y)$ such that $\left\langle e_{1}, \ldots, e_{d}\right\rangle$ forms a $\mathbb{Q}$-basis of $L_{\infty}(Y) \otimes \mathbb{Q}$.

Let $h_{Y} \in \operatorname{Amp}(Y) \cap \mathrm{NS}(Y)$ be an ample class. For any $c \in \mathrm{NS}(Y)$ of square -2 , by Riemann-Roch Theorem, either $c$ or $-c$ is effective. Thus, the intersection pairing between $h_{Y}$ and any class in $\mathrm{NS}(Y)$ of square -2 is not zero. Since $\iota(\mathrm{NS}(X))$ is a sublattice of $\mathrm{NS}(Y)$ of finite index, there exists a sufficiently large integer $N>0$, such that $N e_{1}, \ldots, N e_{d}, N h_{Y} \in \iota(\mathrm{NS}(X))$. Note that the intersection pairing between $N h_{Y}$ and any class in $\iota(\mathrm{NS}(X))$ of square -2 is not zero. Since the ample cone $\operatorname{Amp}(X)$ of $X$ is a standard fundamental domain for the Weyl group $W(\mathrm{NS}(X))$, it follows that there exists $\alpha \in W(\mathrm{NS}(X))$ such that $\alpha\left(\iota^{-1}\left(N h_{Y}\right)\right) \in \operatorname{Amp}(X) \cap \operatorname{NS}(X)$. Then we claim the following:

Lemma 5.3. $\alpha\left(\iota^{-1}\left(N e_{1}\right)\right), \ldots, \alpha\left(\iota^{-1}\left(N e_{d}\right)\right) \in \operatorname{Nef}(X) \cap \operatorname{NS}(X)$, where $\operatorname{Nef}(X)$ is the nef cone of $X$.

Proof. Let $C \subset X$ be a smooth rational curve. Then $\left(\alpha\left(\iota^{-1}\left(N h_{Y}\right)\right),[C]\right)>$ 0 by ampleness of $\alpha\left(\iota^{-1}\left(N h_{Y}\right)\right)$, where $[C]$ denotes the class of $C$ in $\mathrm{NS}(X)$. Then $\left(N h_{Y}, \iota\left(\alpha^{-1}([C])\right)\right)>0$. Since $\iota\left(\alpha^{-1}([C])\right)$ of square -2 , by Riemann-Roch Theorem, $\iota\left(\alpha^{-1}([C])\right)$ is an effective class in $\operatorname{NS}(Y)$. Then $\left(N e_{i}, \iota\left(\alpha^{-1}([C])\right)\right) \geq 0$, for all $i$. Thus, $\left(\alpha\left(\iota^{-1}\left(N e_{i}\right)\right),[C]\right) \geq 0$. So $\alpha\left(\iota^{-1}\left(N e_{i}\right)\right)$ is a nef class in $\operatorname{NS}(X)$.

For any $1 \leq i \leq d$, let $e_{i}^{\prime}$ be the primitive class in $\operatorname{NS}(X)$ such that $\mathbb{R}^{>0} e_{i}^{\prime}=$ $\mathbb{R}^{>0} \alpha\left(\iota^{-1}\left(N e_{i}\right)\right)$. Then $e_{i}^{\prime} \in \mathcal{E}(X)$ according to Piatetsky-Shapiro and Shafarevich [PS71].

Lemma 5.4. $e_{i}^{\prime} \in \mathcal{E}_{\infty}(X)$ for all $i$. 
Proof. Since $e_{i} \in \mathcal{E}_{\infty}(Y)$, by Lemma 2.1, $\operatorname{rk}\left(e_{i}^{\perp}\right)-\operatorname{rk}\left(\left(e_{i}^{\perp}\right)^{(2)}\right)>0$. Since $\operatorname{rk}(\mathrm{NS}(X))=\operatorname{rk}(\mathrm{NS}(Y))$, it follows that $\operatorname{rk}\left(e_{i}^{\perp}\right)=\operatorname{rk}\left(e_{i}^{\perp}\right)$. Since $\mathrm{NS}(X)$ is isometric to a sublattice of $\mathrm{NS}(Y)$, it follows that $\left(e_{i}^{\perp \perp}\right)^{(2)}$ is isometric to a sublattice of $\left(e_{i}^{\perp}\right)^{(2)}$. Then $\operatorname{rk}\left(\left(e_{i}^{\prime \perp}\right)^{(2)}\right) \leq \operatorname{rk}\left(\left(e_{i}^{\perp}\right)^{(2)}\right)$. Thus, $\operatorname{rk}\left(e_{i}^{\prime \perp}\right)-\operatorname{rk}\left(\left(e_{i}^{\prime \perp}\right)^{(2)}\right) \geq \operatorname{rk}\left(e_{i}^{\perp}\right)-\operatorname{rk}\left(\left(e_{i}^{\perp}\right)^{(2)}\right)>0$. Then, again by Lemma $2.1, e_{i}^{\prime} \in \mathcal{E}_{\infty}(X)$.

Since $\operatorname{dim}_{\mathbb{Q}}\left(\mathbb{Q}\left\langle e_{1}, \ldots, e_{d}\right\rangle\right)=d$, it follows that $\operatorname{dim}_{\mathbb{Q}}\left(\mathbb{Q}\left\langle e_{1}^{\prime}, \ldots, e_{d}^{\prime}\right\rangle\right)=d$. Thus, $\operatorname{rk}\left(L_{\infty}(X)\right) \geq d=\operatorname{rk}\left(L_{\infty}(Y)\right)$. Then by Theorem 1.1,

$\max \{$ Salem degree of $f \mid f \in \operatorname{Aut}(X)\} \geq \max \{$ Salem degree of $f \mid f \in \operatorname{Aut}(Y)\}$.

This completes the proof of Theorem 1.2.

Proof of Theorem 1.3. First we consider the cases $p=11$ or $>13$. It is known that the supersingular K3 surface $X(p)$ of Artin invariant one is isomorphic to $\operatorname{Km}\left(E \times_{\mathbb{F}_{p}} E\right)$ for any supersingular elliptic curve $E$ over $\mathbb{F}_{p}([\mathbf{O g u} \mathbf{7 9}]$, [Shi75]). The two natural projections from $E \times_{\mathbb{F}_{p}} E$ to the two factors induce two elliptic fibrations on $X(p)$ with Mordell-Weil rank 4, by the formula of Mordell-Weil rank [Shi90]. Hence $\operatorname{rk}\left(L_{\infty}(X(p))\right) \geq 2$. By Theorem 1.4, $\max \{$ Salem degree of $f \mid f \in \operatorname{Aut}(X(p))\}=22$. By [RS78], the Artin invariant $\sigma(X)$ of $X$ determines $\mathrm{NS}(X)$ up to isometry. Moreover, by explicit classification of the lattices $\mathrm{NS}(X)$ and $\mathrm{NS}(X(p))$ in $[\mathbf{R S 7 8}], \mathrm{NS}(X)$ is isometric to a sublattice of NS $(X(p))$ (cf. [Li15, proof of Proposition 3.9]). Then by Theorem 1.2, $\max \{$ Salem degree of $f \mid f \in \operatorname{Aut}(X)\} \geq \max \{$ Salem degree of $f \mid f \in \operatorname{Aut}(X(p))\}=22$. Then the maximal Salem degree of automorphisms of $X$ is 22 since the Picard number of $X$ is 22 . Thus, when $p=11$ or $>13$, there exists $f \in \operatorname{Aut}(X)$ the entropy of which is the logarithm of a Salem number of degree 22. The case $p=3$ is proved by [EO15] and [Sh16]. The cases $p=5,7,13$ are proved by [Sh16] (the case $p=7$ is also covered by [Br15]). This completes the proof of Theorem 1.3.

\section{The exceptional sublattice, elliptic fibrations and Salem numbers.}

In this section, we discuss some relationships among the exceptional sublattice, elliptic fibration and the maximal Salem degree of automorphisms of K3 surfaces.

Following [Ni14], for a K3 surface $X$, we define the exceptional sublattice of the Néron-Severi group $\mathrm{NS}(X)$ by

$E(\mathrm{NS}(X)):=\{x \in \mathrm{NS}(X) \mid$ the orbit $\operatorname{Aut}(X)(x)$ of $x$ in $\mathrm{NS}(X)$ is finite $\}$.

Clearly, $E(\mathrm{NS}(X))$ is a primitive sublattice of $\mathrm{NS}(X)$. For a sublattice $F \subset \mathrm{NS}(X)$ we denote by $F_{p r}$ the primitive sublattice $F_{p r}=\mathrm{NS}(X) \cap(F \otimes \mathbb{Q}) \subset \mathrm{NS}(X) \otimes \mathbb{Q}$ generated by $F$.

TheOrem 6.1 ([Ni14, Theorem 4.1]). Let $X$ be a K3 surface defined over an algebraically closed field $k$ of characteristic $p \neq 2,3$. Suppose $X$ has at least two elliptic fibrations with infinite automorphism groups. Then 


$$
E(\mathrm{NS}(X))=\bigcap_{e \in \mathcal{E}_{\infty}(X)}\left(e^{\perp}\right)_{p r}^{(2)}=L_{\infty}(X)^{\perp}
$$

Proof. Since $X$ has at least two elliptic fibrations with infinite automorphism groups, it follows that the sublattice $L_{\infty}(X)^{\perp} \subset \mathrm{NS}(X)$ is negative definite. Then $L_{\infty}(X)^{\perp} \subset E(\mathrm{NS}(X))$ since $L_{\infty}(X)^{\perp}$ is Aut $(X)$-stable and $\mathrm{O}\left(L_{\infty}(X)^{\perp}\right)$ is a finite group. On the other hand, by [Ni14, Theorem 4.1], $E(\mathrm{NS}(X))=\bigcap_{e \in \mathcal{E}_{\infty}(X)}\left(e^{\perp}\right)_{p r}^{(2)} \subset L_{\infty}(X)^{\perp}$. Thus, $E(\mathrm{NS}(X))=\bigcap_{e \in \mathcal{E}_{\infty}(X)}\left(e^{\perp}\right)_{p r}^{(2)}=L_{\infty}(X)^{\perp}$.

For a K3 surface with even Picard number, we collect various methods to check whether it has an automorphism of maximal possible Salem degree:

TheOREm 6.2. Let $X$ be a K3 surface defined over an algebraically closed field $k$ of characteristic $p \neq 2,3$. Suppose $X$ has even Picard number $\rho(X) \geq 4$, and suppose $X$ has at least one elliptic fibration with infinite automorphism group. Then the following statements are equivalent to each other:

1) Any $\operatorname{Aut}(X)$-stable $\mathbb{R}$-linear subspace of $\mathrm{NS}(X) \otimes \mathbb{R}$ is either $\{0\}$ or $\mathrm{NS}(X) \otimes \mathbb{R}$;

2) Any $\operatorname{Aut}(X)$-stable $\mathbb{Q}$-linear subspace of $\mathrm{NS}(X) \otimes \mathbb{Q}$ is either $\{0\}$ or $\mathrm{NS}(X) \otimes \mathbb{Q}$;

3) $\mathbb{Q}\left\langle\mathcal{E}_{\infty}(X)\right\rangle=\mathrm{NS}(X) \otimes \mathbb{Q}$;

4) There exists $f \in \operatorname{Aut}(X)$ such that the Salem degree of $f$ is $\rho(X)$;

5) $\bigcap_{e \in \mathcal{E}_{\infty}(X)}\left(e^{\perp}\right)_{p r}^{(2)}=\{0\}$;

6) $E(\mathrm{NS}(X))=\{0\}$.

Proof. 1) $\Longrightarrow 2$ 2) $\Longrightarrow 3$ ): Trivial.

3) $\Longrightarrow 1$ ): By Theorem 5.1.

3) $\Longrightarrow$ 4): By Theorem 1.1.

$4) \Longrightarrow 3)$ : Suppose $X$ has an automorphism $f \in \operatorname{Aut}(X)$ whose entropy is the logarithm of a Salem number of degree $\rho(X)$. Then $\mathrm{NS}(X) \otimes \mathbb{Q}$ has no non-trivial $f$-stable $\mathbb{Q}$-subspace (cf. [Mc02, Proof of Theorem 3.4]). On the other hand, $\mathbb{Q}\left\langle\mathcal{E}_{\infty}(X)\right\rangle$ is clearly $f$-stable. Therefore, $\mathbb{Q}\left\langle\mathcal{E}_{\infty}(X)\right\rangle=\mathrm{NS}(X) \otimes \mathbb{Q}$.

$5) \Longleftrightarrow 6)$ : By Theorem 6.1.

$2) \Longrightarrow 6)$ : By definition, $E(\operatorname{NS}(X)) \otimes \mathbb{Q}$ is clearly an Aut $(X)$-stable $\mathbb{Q}$-linear subspace of $\mathrm{NS}(X) \otimes \mathbb{Q}$. Then either $E(\mathrm{NS}(X))=\{0\}$ or $\mathrm{NS}(X)$. If $E(\mathrm{NS}(X))=\mathrm{NS}(X)$, then $\operatorname{Aut}(X)$ must be a finite group, a contradiction to the assumption $\mathcal{E}_{\infty}(X) \neq \emptyset$. Therefore, $E(\mathrm{NS}(X))=\{0\}$.

$6) \Longrightarrow 3)$ : Since $E(\operatorname{NS}(X))=\{0\}$, it follows that $\mathcal{E}_{\infty}(X)$ is an infinite set. Then the sublattice $L_{\infty}(X)^{\perp} \subset \mathrm{NS}(X)$ is negative definite and $\mathrm{O}\left(L_{\infty}(X)^{\perp}\right)$ is a finite group. Obviously, $L_{\infty}(X)^{\perp}$ is $\operatorname{Aut}(X)$-stable. Then $L_{\infty}(X)^{\perp} \subset E(\mathrm{NS}(X))=\{0\}$. Thus, $\mathbb{Q}\left\langle\mathcal{E}_{\infty}(X)\right\rangle=\mathrm{NS}(X) \otimes \mathbb{Q}$.

REMARK 6.3. $\quad$ i) By Theroems 1.3 and $6.2, E(\mathrm{NS}(X))=\{0\}$ for any supersingular K3 surface $X$ in odd characteristic. 
ii) Let $X$ be the Kummer surface of the Jacobian of a very general curve $C$ of genus 2 over an algebraically closed field of characteristic 0 . Then $X$ is a K3 surface of Picard number 17. Elliptic fibrations with a section on $X$ are completely classified in [Ku14]. Using elliptic fibrations explicitly given in the table on $[\mathbf{K u 1 4}$, pp. 609 $610]$, one can easily show that $\operatorname{rk}\left(L_{\infty}(X)\right)=17$. Thus, by Theorem $1.1, X$ has an automorphism the entropy of which is the logarithm of a Salem number of degree 16 .

iii) Let $X$ be the Kummer surface $\operatorname{Km}(E \times F)$, where complex elliptic curves $E$ and $F$ are not isogenous. Then $X$ is a complex K3 surface with Picard number 18. Thanks to complete classification of elliptic fibrations with a section on $X([\mathbf{O g} 89])$, one can show that $\operatorname{rk}\left(L_{\infty}(X)\right) \geq 10$. Thus, by Theorem 1.1, $\max \{$ Salem degree of $f \mid f \in \operatorname{Aut}(X)\} \geq 10$. (See $[$ Og16] for a higher dimensional application.) On the other hand, the exceptional lattice $E(\mathrm{NS}(X))$ is negative definite and, by $[\mathbf{O g 8 9}$, Lemma 1.4], contains a sublattice of rank 8. Then the Salem degree of any automorphism of $X$ must be $\leq 18-8=10$. Therefore, we conclude that $\max \{$ Salem degree of $f \mid f \in \operatorname{Aut}(X)\}=10$. Note that $\operatorname{NS}(X)$ is a 2-elementary lattice (for similar examples of K3 surfaces $Y$ with $E(\mathrm{NS}(Y)) \neq\{0\}$, see [Ni99, Section 3])

REMARK 6.4. Let $\mathcal{S E K} 3^{\prime}$ be the set of all even hyperbolic lattices $S$ of $\operatorname{rk}(S) \geq 3$ with the following property: There exists a K3 surface $X$ defined over an algebraically closed field of characteristic $\neq 2,3$ such that $S$ is isometric to $\operatorname{NS}(X)$, and $E(\operatorname{NS}(X)) \neq$ $\{0\}$. By [Ni14, Theorem 4.4] and [LM11, Theorem 6.1], the set $\mathcal{S} E K 3^{\prime}$ is finite (note that $\mathcal{S E K 3} 3^{\prime}$ defined here is a subset of the set $\mathcal{S E K 3}$ defined by [Ni14, Definition 4.5]). A consequence of finiteness of $\mathcal{S E K} 3^{\prime}$ is the following: There are only finitely many singular K3 surfaces $X$ (i.e., complex K3 surfaces with Picard number 20) such that $\max \{$ Salem degree of $f \mid f \in \operatorname{Aut}(X)\} \leq 18$. It would be interesting to find the finite set of Néron-Severi groups $\mathcal{S E K 3}{ }^{\prime}$ (or even $\mathcal{S E K} 3$ ) of K3 surfaces.

\section{References}

[Ar74] M. Artin, Supersingular K3 surfaces, Ann. Sci. École Norm. Sup. (4), 7 (1974), 543-567.

[BC16] J. Blanc and S. Cantat, Dynamical degrees of birational transformations of projective surfaces, J. Amer. Math. Soc., 29 (2016), 415-471.

[Br15] S. Brandhorst, Dynamics on supersingular K3 surfaces and automorphisms of Salem degree 22, http://arxiv.org/pdf/1507.02092v1.pdf.

[Br16] S. Brandhorst, Automorphisms of Salem degree 22 on supersingular K3 surfaces of higher Artin invariant - a short note, https://arxiv.org/pdf/1609.02348v1.pdf.

[BG16] S. Brandhorst and V. González-Alonso, Automorphisms of minimal entropy on supersingular K3 surfaces, https://arxiv.org/pdf/1609.02716v1.pdf.

[ES13] H. Esnault and V. Srinivas, Algebraic versus topological entropy for surfaces over finite fields, Osaka J. Math., 50 (2013), 827-846.

[EO15] H. Esnault and K. Oguiso, Non-liftability of automorphism groups of a K3 surface in positive characteristic, Math. Ann., 363 (2015), 1187-1206.

[EOY16] H. Esnault, K. Oguiso and X. Yu, Automorphisms of elliptic K3 surfaces and Salem numbers of maximal degree, Algebraic Geometry, 3 (2016), 496-507.

[Ku14] A. Kumar, Elliptic fibrations on a generic Jacobian Kummer surface, J. Algebraic Geometry, 23 (2014), 599-667. 
[LM11] M. Lieblich and D. Maulik, A note on the cone conjecture for K3 surfaces in positive characteristic, http://arxiv.org/pdf/1102.3377v3.pdf.

[Li15] C. Liedtke, Supersingular K3 surfaces are unirational, Inventiones mathematicae, 200 (2015), 979-1014.

[Mc02] C. T. McMullen, Dynamics on K3 surfaces: Salem numbers and Siegel disks, J. Reine Angew. Math., 545 (2002), 201-233.

[Ni83] V. V. Nikulin, Factor groups of groups of automorphisms of hyperbolic forms with respect to subgroups generated by 2-reflections, Algebrogeometric applications, J. Math. Sci., 22 (1983), 1401-1475.

[Ni99] V. V. Nikulin, K3 surfaces with interesting groups of automorphisms, J. Math. Sci., 95 (1999), 2028-2048.

[Ni14] V. V. Nikulin, Elliptic fibrations on K3 surfaces, Proc. Edinb. Math. Soc. (2), 57 (2014), $253-267$.

[Og89] K. Oguiso, On Jacobian fibrations on the Kummer surfaces of the product of non-isogenous elliptic curves, J. Math. Soc. Japan, 41 (1989), 651-680.

[Og07] K. Oguiso, Automorphisms of hyperkähler manifolds in the view of topological entropy, Algebraic geometry, Contemp. Math., 422, Amer. Math. Soc., Providence, RI, 2007, 173-185.

[Og09] K. Oguiso, Mordell-Weil groups of a hyperkähler manifold-a question of F. Campana, Publ. RIMS, 44 (2009), 495-506.

[Og10] K. Oguiso, Salem polynomials and the bimeromorphic automorphism group of a hyper-Kähler manifold, Selected papers on analysis and differential equations, Amer. Math. Soc. Transl. Ser., 230 (2010), 201-227.

[Og16] K. Oguiso, Pisot units, Salem numbers and higher dimensional projective manifolds with primitive automorphisms of positive entropy, https://arxiv.org/pdf/1608.03122v3.pdf.

[Ogu79] A. Ogus, Supersingular K3 crystals, Journées de Géométrie Algébrique de Rennes, Astérisque, 64 (1979), 3-86.

[Ogu83] A. Ogus, A crystalline Torelli theorem for supersingular K3 surfaces, Progr. Math., 36 (1983), 361-394.

[PS71] I. I. Piatetsky-Shapiro and I. R. Shafarevich, A Torelli theorem for algebraic surfaces of type K3, Math. USSR-Izv., 5 (1971), 547-588.

[RS78] A. N. Rudakov and I. R. Shafarevich, Supersingular K3 surfaces over fields of characteristic 2, Izv. Akad. Nauk SSSR, 42 (1978), 848-869, Math. USSR-Izv., 13 (1979), 147-165.

[Sch15] M. Schütt, Dynamics on supersingular K3 surfaces, http://arxiv.org/pdf/1502.06923v2.pdf.

[Sh16] I. Shimada, Automorphisms of supersingular K3 surfaces and Salem polynomials, Exp. Math., 25 (2016), 389-398.

[Shi75] T. Shioda, Algebraic cycles on certain K3 surfaces in characteristic $p$, Manifolds-Tokyo 1973, Proc. Internat. Conf., Tokyo, 1973, Univ. Tokyo Press, Tokyo, 1975, 357-364.

[Shi90] T. Shioda, On the Mordell-Weil lattices, Comm. Math. Univ. St. Paul, 39 (1990), 211-240.

\section{Xun Yu}

Center for Applied Mathematics Tianjin University

92 Weijin Road, Nankai District

Tianjin 300072, China

E-mail: xunyu@tju.edu.cn 